\title{
Virus induced PhFTRv gene silencing results in yellow-green leaves and reduced cold tolerance in petunia
}

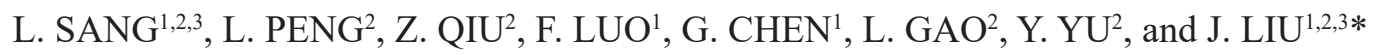 \\ College of Horticulture, South China Agricultural University, Guangzhou 510642, P.R. China ${ }^{1}$ \\ College of Forestry and Landscape Architecture, South China Agricultural University, \\ Guangzhou 510642, China ${ }^{2}$ \\ Lingnan Guangdong Laboratory of Modern Agriculture, Guangzhou, 510642, P.R. China ${ }^{3}$
}

\begin{abstract}
Ferredoxin-thioredoxin reductase (FTR) is an iron-sulfur protein that supplies electrons from photochemically reduced ferredoxin (Fd) to thioredoxin (Trx) in the ferredoxin/thioredoxin system in chloroplasts. The FTR is a heterodimer with a variable subunit (FTRv) and a catalytic subunit (FTRc). The function of FTRv is not well known. In petunia (Petunia hybrida), FTRv is a single-copy gene, which is named PhFTRv. In this study, the spatio-temporal expression of PhFTRv in petunia was analyzed, and PhFTRv transcription was found to be high in leaves and stems. A tobacco rattle virus gene silencing was used in this study. Virus induced gene silencing-mediated PhFTRv silencing resulted in large yellow-green leaves, delayed flowering, and reduced cold tolerance in petunia plants.
\end{abstract}

Additional key words: flowering, chloroplasts, chlorophyll, leaf development, Petunia hybrida, photosynthesis, temperature stress.

\section{Introduction}

Thioredoxins (Trxs) serve as a key factor in the redox system that regulates the activity of a series of target proteins through dithiol-disulfide exchange reactions in plants. Chloroplasts, which serve as the site of photosynthesis, contain at least two systems of thiol-disulfide reductases that function in the control of redox-dependent protein stability and/or activity (Geigenberger and Fernie 2014, Nikkanen et al. 2016). The ferredoxin/thioredoxin $(\mathrm{Fd} / \mathrm{Trx})$ system plays important roles in chloroplasts (Buchanan et al. 2002). Ferredoxin-thioredoxin reductase (FTR), an iron-sulfur protein, supplies electrons from photochemically reduced ferredoxin to thioredoxin in chloroplasts under light conditions and plays an important role in the $\mathrm{Fd} / \mathrm{Trx}$ regulatory chain (Dai et al. 2000). The FTR could efficiently reduce chloroplast f- and m-type Trxs. The reduced thioredoxins activate target enzymes, thereby activating anabolic pathways in metabolism (Dai et al. 2000). In addition, plastids contain an NADPHdependent thioredoxin reductase C (NTRC), which is a key redox-mediator protein activated by NADPH both under light and dark conditions and responsible for regulatory functions that is able to catalyse thiol-disulfide exchange reactions in target enzymes (Serrato et al. 2004, Yoshida and Hisabori 2017). Both FTR and NTRC act as key regulators of ATP synthase by regulating the $\gamma$ subunit under limited light conditions (Luo et al. 2012, Yoshida et al. 2013). In plants, studies of the Trx system have mainly focused on stress resistance (Lim et al. 2010, Dietz and Pfannschmidt 2011, Chae et al. 2013, Belin et al. 2015).

Ferredoxin-thioredoxin reductase is a heterodimer with a variable subunit (FTRv) and a catalytic subunit (FTRc). In plants, both subunits are encoded in the nucleus (Schurmann and Jacquot 2000). FTRc contains a redoxactive disulfide and a [4Fe-4S] centre (Schurmann and Jacquot 2000). FTR has been crystallized, and its structure has been solved. The variable subunit has an open $\beta$-barrel structure, and the catalytic subunit is essentially $\alpha$-helical (Dai et al. 2000). In Solanum lycopersicum, SlFTRc silencing induces the expression of pathogenesis-related genes and pathogen resistance, and SIFTRc acts as a regulator of programmed cell death (PCD) and pathogen resistance (Lim et al. 2010). In A. thaliana, the young inner leaves of FTR $c$-silenced plants exhibit a sectorial chlorotic leaf phenotype and chloroplasts in FTRc-silenced leaves are not fully developed (Wang et al. 2014).

In $A$. thaliana, there are two FTRv isoforms, AtFTRA1

Submitted 28 June 2020, last revision 27 September 2020, accepted 12 October 2020.

Abbreviations: $\mathrm{c}_{\mathrm{i}}$ - internal $\mathrm{CO}_{2}$ concentration; Fd - ferredoxin; FTR - ferredoxin-thioredoxin reductase; gs - stomatal conductance; NTRC - NADPH-dependent thioredoxin reductase C; $P_{N}$ - net photosynthetic rate; TRV - tobacco rattle virus; Trx - thioredoxin; VIGS - virus induced gene silencing.

Acknowledgements: This research was supported by the National Natural Science Foundation of China (31770737, 31870692 and 31661143047) and the National Key Research and Development Plan (2018YFD100015).

* Corresponding author; e-mail: juanxuliu@scau.edu.cn 
and AtFTRA2, and only single AtFTRA1 mutant plants exhibit subtle developmental changes when compared to wild-type plants (Keryer et al. 2004). Plants with disrupted AtFTRA were found to be significantly more sensitive to oxidative stress (Keryer et al. 2004). There is only one FTRv gene in some species of Solanaceae, such as petunia (Petunia hybrida), tomato, and pepper. Thus, it is necessary to clearly define the biological function of FTRv.

The aim of this study was to analyze the spatiotemporal expression of PhFTRv in petunia as affected by a low temperature and virus induced gene silencing.

\section{Materials and methods}

Plant materials and growth conditions: Petunia (Petunia hybrida J.D. Hooker Vilmorin) cv. Ultra was grown in a culture chamber with a 14-h photoperiod, day/night temperatures of $24 / 12{ }^{\circ} \mathrm{C}$, a relative humidity of $60-70 \%$, and an irradiance of $250 \mu \mathrm{mol} \mathrm{m}^{-2} \mathrm{~s}^{-1}$. Flower, leaf, stem, and root tissues were collected from 4- to 8-week-old petunia plants. Samples from the corollas were harvested at anthesis.

RNA extraction and real-time quantitative PCR assays: Total RNA was extracted and reverse-transcribed according to previous methods (Liu et al. 2011). Total RNA was extracted using TRIzol according to the manufacturer's instructions and treated with DNase using amplification grade DNase (Sangon Biotech, Guangzhou, China). The sequences of PhFTRv were obtained from the petunia genome (https://solgenomics.net/organism/ Petunia axillaris/genome). The kit (TSE202, Tsingke, Guangzhou, China) was used for quantitative PCR (qPCR) analysis. The $\mathrm{qPCR}$ procedure is set according to the instructions of the kit: $95^{\circ} \mathrm{C} / 60 \mathrm{~s}, 95^{\circ} \mathrm{C} / 10 \mathrm{~s}, 55^{\circ} \mathrm{C} / 5 \mathrm{~s}$, $72{ }^{\circ} \mathrm{C} / 10$ s, 40 cycles. The petunia Actin (accession No. FN014209) was used as internal reference gene to quantify the cDNA abundances. Melting curve analysis is referred to Chang et al. (2016), data analysis is carried out by the $2^{-\triangle \Delta C T}$ method (Livak and Schmittgen 2001). The sequences of all primers used for qPCR analysis are in Table 1 Suppl.

Sequence analysis: Alignments were performed using $D N A M A N$ software, and a phylogenetic tree was generated using ETE3 v3.1.1 software (https://www.genome.jp/ tools-bin/ete). Searches of nucleotide and translated amino acid sequences were performed using the National Center for Biotechnology Information (NCBI) BLAST network server (https://blast.ncbi.nlm.nih.gov/Blast.cgi).

Virus induced gene silencing (VIGS) assay: A tobacco rattle virus (TRV)-based VIGS system described previously was used to silence the PhFTRv gene (Chen et al. 2017). The TRV based cDNA clone was placed between the CaMV 35S promoter with duplicated enhancer region and the nopaline synthase terminator in a T-DNA vector. The pTRV1 vector (AF406990, GenBank) includes RNA1 part of T-DNA construct and pTRV2 vector (AF406991,
GenBank) includes RNA2 part of T-DNA construct, pTRV1 and pTRV2 must be used at the same time in an infection (Liu et al. 2002). To construct the pTRV2-FTRv vectors, the coding region of PhFTR $v$ was amplified by real time qPCR from a petunia cDNA library with forward primer 5'CGGGATCCCTAACAACCCTTTATCCC3' and reverse primer 5'CGGAATTCATACTGTTTCAATGTCCC3'. The method of VIGS was described previously (Chen et al. 2017). The pTRV2 vector or its derivatives and the pTRV1 vector were simultaneously introduced into Agrobacterium tumefaciens L. strain EHA105. The A. tumefaciens strains containing plasmid constructs were grown overnight at $28^{\circ} \mathrm{C}$ in Luria-Bertani medium with $50 \mathrm{mg} \mathrm{dm}^{-3}$ kanamycin and $200 \mathrm{mM}$ acetosyringone. The cells were harvested and resuspended in sterile $10 \mathrm{mM} \mathrm{MES}$ and $10 \mathrm{mM} \mathrm{MgCl}_{2}$ buffer with $200 \mu \mathrm{M}$ acetosyringone. The bacteria containing pTRV1 were mixed with bacteria containing the pTRV2 derivatives in a 1:1 ratio, and the absorbances $\left(\mathrm{A}_{600}\right)$ were measured before the infiltration of petunia plantlets. The expressions of PhFTR $v$ and PhFTRc in $P h F T R v$-silenced plants were determined by qPCR.

Photosynthesis measurements and analyses: Photosynthesis in leaves was measured according to a method described in a previous study (He et al. 2018). Three healthy leaves were selected from each plant from among the $3^{\text {rd }}$ to $4^{\text {th }}$ leaves on the middle-upper branches. A GFS-3000 portable photosynthetic system (Walz, Effeltrich, Germany) was used to monitor the response of photosynthesis to irradiance in the selected leaves at 11:00 every day. We then obtained values for net photosynthetic rate $\left(\mathrm{P}_{\mathrm{N}}\right)$, stomatal conductance $\left(\mathrm{g}_{\mathrm{s}}\right)$, and intercellular $\mathrm{CO}_{2}$ concentration $\left(\mathrm{c}_{\mathrm{i}}\right)$. The measurements were repeated three times for each leaf, and three biological replicates were performed.

Determination of chlorophyll content: Chlorophyll content was determined according to our previously described protocol (Liu et al. 2019). Approximately $1.5 \mathrm{~g}$ of leaves were harvested and freeze-dried, and three replicate methanol extractions were prepared. The absorbance of the solution was measured at 646.8 , 663.2, and $470.0 \mathrm{~nm}$ and compared to that of the solvent (methanol) blank. The content of total chlorophyll was measured with a spectrophotometer and calculated according to Lichtenthaler (1987).

Statistical analysis: The data were analyzed using SPSS v. 11.09 (IBM Corporation, Armonk, NY, USA) and are presented as the means of three replicates. A least significant difference (LSD) test was used to analyze the differences between the means. The significance level was set at $\alpha<0.05$.

\section{Results}

To obtain the sequences of the PhFTRv cDNAs in petunia, the amino acid sequence of the AtFTRv gene from $A$. thaliana (AtFTRA1, locus BAB09560) (Keryer 
et al. 2004) was used as a query for BLAST searches of the Petunia axillaris draft genome sequence (v1.6.2); only one FTRv sequence from petunia was recovered. PhFTRv was predicted to encode a protein containing 155 amino acids. The multiple sequence alignments of petunia PhFTRv, A. thaliana AtFTRA1 (AT5G23440) and AtFTRA2 (At5g08410), Solanum lycopersicum SlFTRV (XP 004229646), Nicotiana tomentosiformis NtFTRv (XP_016508304), Oryza sativa OsFTRv1 (XP_015634256) and OsFTRv2 (XP_015623472) are presented in Fig. 1A. The protein alignment showed that the carboxyl-terminus of FTRv in different species showed high sequence similarity, while the sequence similarity of the amino-terminus was very low (Fig. 1A). In addition, FTRv contained a conserved EDEFE motif in its carboxyl-terminus. PhFTRv shared 78.1 and $74.5 \%$ amino acid sequence similarity with NtFTRv and SlFTRv, respectively (Table 2 Suppl.).

To elucidate the evolutionary relationships among FTRv proteins in dicot and monocot species with genome sequences that have not been published, we examined FTRv-like sequences in 14 dicot and 4 monocot species that were randomly selected, and a phylogenetic tree was generated using $M E G A$ software with amino acid sequences derived from GenBank (Fig. 1B, Fig. 1 Suppl.). The phylogenetic tree showed that the FTRv family in dicot species included one or two members, and three members were found only in Brassica rapa. In the monocot species, the FTRv family generally included two members, and only three members were found in Ananas comosus (Fig. 1 Suppl.).

The expression of PhFTRv was examined in different plant organs by real time qPCR. The PhFTR $v$ transcription was high in leaves and stems and low in roots (Fig. 2A). A previous study demonstrated the role of $S$. lycopersicum SIFTRc in plant stress responses, and the lesion mimic phenotypes related to defense in $S$. lycopersicum slftrc mutants are dependent on temperature (Lim et al. 2010). The effects of different temperatures on the expression of PhFTRv were examined, and the results showed that low temperatures increased the transcription of PhFTRv (Fig. 2B).

Four to five weeks old petunia seedlings were infected with a mixture of Agrobacterium transformed with pTRV2-PhFTRv or pTRV2 vectors and Agrobacterium transformed with the pTRV1 vector. The expressions of PhFTRv and PhFTRc (Peaxi162Scf00129g00821.1) were examined in plants inoculated with Agrobacterium containing pTRV2-FTRv and pTRV2 by real time qPCR. Although PhFTRv transcripts were detected in the PhFTRv-silenced plants, the expression of PhFTRv was significantly reduced compared with control plants that were inoculated with Agrobacterium containing the empty pTRV2 vector (Fig. 2C). The expression of PhFTRc was not affected by PhFTRv silencing (Fig. 2D).

The newly emerged leaves of plants infected with Agrobacterium containing pTRV2-PhFTRv exhibited a yellow-green leaf phenotype, while those infected by the

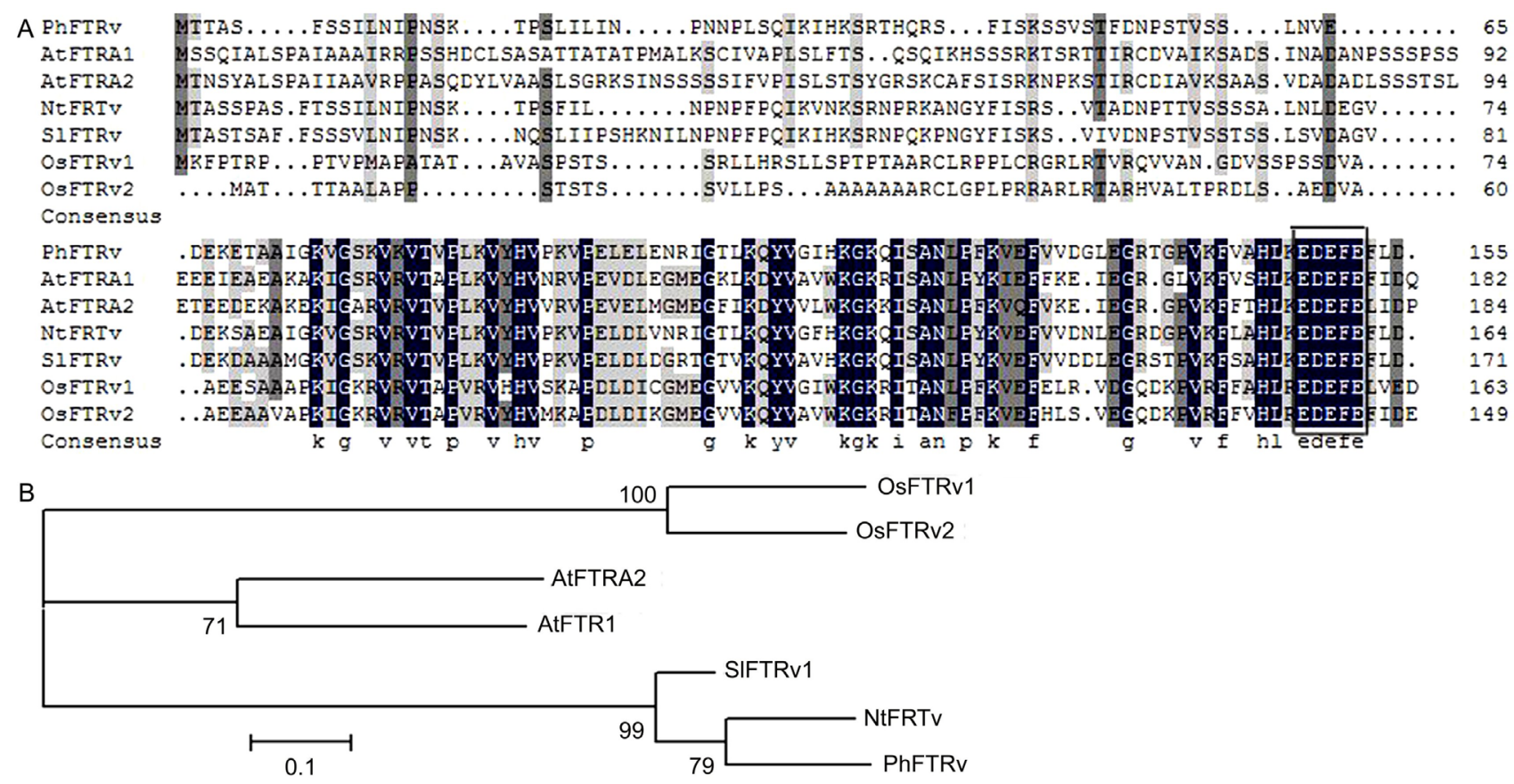

Fig. 1. Predicted amino acid sequence alignments and neighbour-joining trees of ferredoxin-thioredoxin reductase variable subunit (FTRv). A - predicted amino acid sequence alignments of petunia PhFTRv with Arabidopsis thaliana AtFTRA1 (BAB09560) and AtFTRA2 (At5g08410), Solanum lycopersicum SIFTRv (XP_004229646), Nicotiana tomentosiformis NtFTRv (XP_016508304), and Oryza sativa OsFTRv1 (XP 015634256) and OsFTRv2 (XP_015623472), respectively. Conserved residues are shaded in black. Grey shading indicates similar residues in six out of seven of the sequences. Light grey shading indicates similar residues in four out of seven of the sequences. The carboxyl-terminal EDEFE is boxed. $B$ - neighbour-joining trees among proteins encoded by the FTRv-like genes using the ETE3 v3.1.1 software (https://www.genome.jp/tools-bin/ete). 

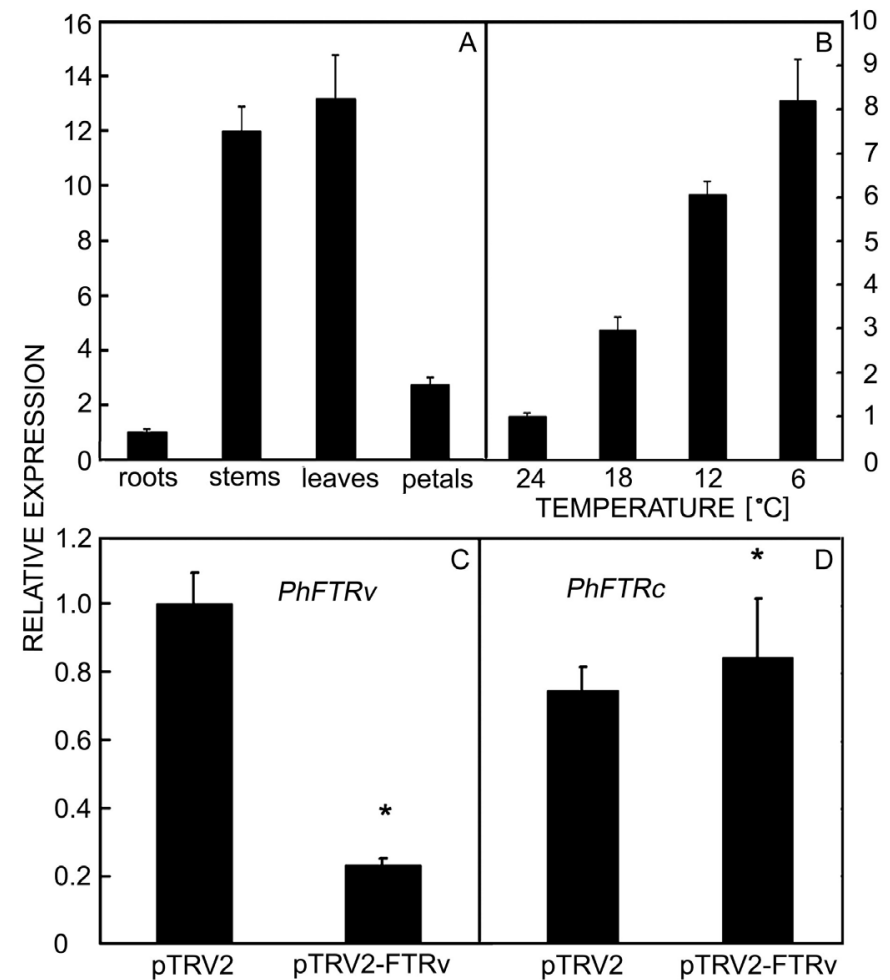

Fig. 2. The expression patterns of Petunia hybrida ferredoxin-thioredoxin reductase variable subunit (PhFTRv) and Petunia hybrida ferredoxin-thioredoxin reductase catalytic subunit (PhFTRc) determined by real time quantitative PCR (qPCR). $A$ and $B$ - the expression patterns of PhFTRv in different organs $(A)$ and in leaves $(B)$ in response to different temperatures. $C$ and $D$ - the effects of pTRV2PhFTRv treatment on the expression of PhFTRv $(C)$ and PhFTRc $(D)$ in the fifth leaves under the buds from five-week-old plants as determined by real time qPCR. Actin was used as an internal reference gene to quantify the cDNA abundance. The relative expressions are presented as fold-change values. Means \pm SDs, $n=3{ }^{*}$ - significant differences at $P<0.05$.


Fig. 3. The phenotypes of Petunia hybrida ferredoxin-thioredoxin reductase variable subunit (PhFTRv)-silenced plants in response to different temperatures. $A$ - $D$ - different leaf colors in four-week-old plants infected with Agrobacterium with plasmid tobacco rattle virus 2 (pTRV2) (A and $C$ ) or $p T R V 2-P h F T R v(B$ and $D)$ and grown at $24{ }^{\circ} \mathrm{C} ; E-H$ - different leaf colors in four-week-old plants with $p T R V 2$ $\left(E\right.$ and $G$ ) or $p T R V 2-P h F T R v\left(F\right.$ and $H$ ) grown at $12{ }^{\circ} \mathrm{C}$; I-J - leaves of control $(I)$ and $P h F T R v$-silenced $(J)$ plants grown at $24{ }^{\circ} \mathrm{C}$, and leaves of control $(K)$ and PhFTRv-silenced $(L)$ plants grown at $12{ }^{\circ} \mathrm{C} ; M-N$ - differences in flowering in seven-week-old plants with pTRV2 $(M)$ and $p T R V 2-\operatorname{PhFTRv}(N)$ grown at $24^{\circ} \mathrm{C}$. 
Table 1. Effects of Petunia hybrida ferredoxin-thioredoxin reductase variable subunit silencing on plant growth. Means \pm SEs from 15 to 20 samples. Statistical analysis was performed using Student's $t$-test; asterisks mean significant differences at $P<0.05$. pTRV2 plasmid tobacco rattle virus 2, pTRV2-FTRv - pTRV2- ferredoxin-thioredoxin reductase variable subunit.

\begin{tabular}{lcrl}
\hline & pTRV2 (control) & pTRV2-FTRv & pTRV2-FTRv/pTRV2 [\%] \\
\hline Length of leaf $[\mathrm{cm}]$ & $4.38 \pm 0.33$ & $5.39 \pm 0.43^{*}$ & 123.1 \\
Width of leaf $[\mathrm{cm}]$ & $2.52 \pm 0.21$ & $3.14 \pm 0.26^{*}$ & 124.6 \\
Diameter of abaxial epidermal cells of leaf $[\mu \mathrm{m}]$ & $91.28 \pm 5.29$ & $113.39 \pm 6.52^{*}$ & 122.3 \\
\hline
\end{tabular}



Fig. 4. Effects of Petunia hybrida ferredoxin-thioredoxin reductase variable subunit silencing on the total chlorophyll content in leaf mesophyll cells from five-week-old plants. pTRV2 - plasmid tobacco rattle virus 2, pTRV2-FTRv - plasmid tobacco rattle virus 2 -ferredoxin-thioredoxin reductase variable subunit. Means \pm SDs, $n=3, *$ - significant differences at $P<0.05$.

Agrobacterium containing pTRV2 (control plants) showed a green leaf phenotype (Fig. $3 A-D)$. The leaves of plants containing pTRV2-PhFTRv maintained a yellow-green leaf phenotype until they became old. Compared with the control plants, the size of the leaves in the plants containing pTRV2-PhFTRv was larger (Fig. 3I,J, Table 1), the content of chlorophyll in the leaves was significantly reduced (Fig. 4), and the epidermal cells on the abaxial surface were larger (Fig. 2 Suppl., Table 1).

The effect of PhFTRv silencing on leaf physiological parameters was further analyzed. The internal $\mathrm{CO}_{2}$ concentration increased significantly in PhFTRv-silenced leaves, while PhFTRv silencing significantly decreased $\mathrm{P}_{\mathrm{N}}$ and $g_{s}(\alpha=0.05)$ (Fig. 5).

In variegated mutants, most leaves have pale green areas with abnormally small mesophyll cells and reduced numbers of chloroplasts (Sakamoto et al. 2003). To determine the effect of PhFTRv suppression on the number of chloroplasts, the protoplasts in mature leaves were extracted and observed under an optical microscope. The numbers of chloroplasts in mesophyll cells in PhFTRvsilenced plants were reduced compared with those in control plants (Fig. 2 Suppl.).

In addition, a delay of two weeks for the emergence of the floral buds was observed in PhFTRv-silenced plants, and PhFTRv silencing delayed flowering by approximately ten days (Fig. 3M,N). Moreover, even when blooming, the number of flowers in PhFTRv-silenced plants was less than in control plants.

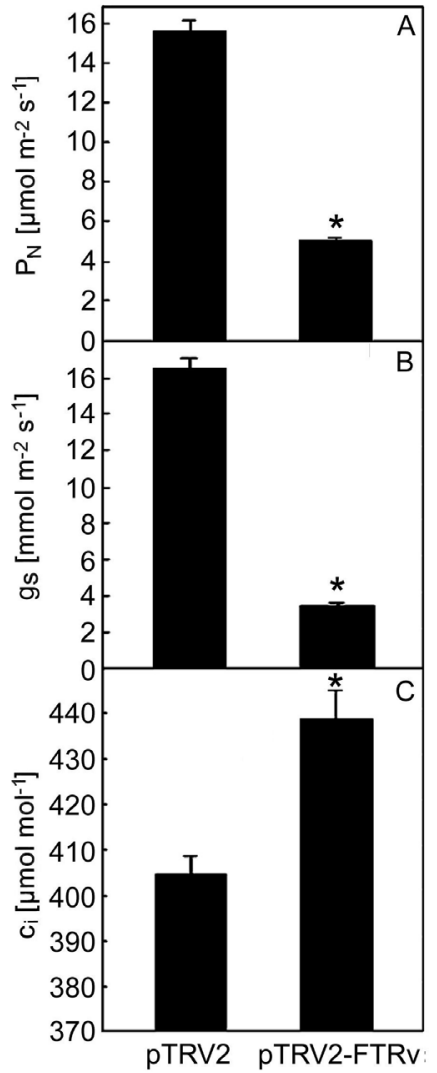

Fig. 5. Effect of Petunia hybrida ferredoxin-thioredoxin reductase variable subunit silencing on photosynthesis characteristics in leaves of petunia grown in pots in a greenhouse. $\mathrm{P}_{\mathrm{N}}$ - net photosynthetic rate, $\mathrm{g}_{\mathrm{s}}$ - stomatal conductance, $\mathrm{c}_{\mathrm{i}}$ - intercellular $\mathrm{CO}_{2}$ concentration. pTRV2 - plasmid tobacco rattle virus 2 , pTRV2-FTRv - plasmid tobacco rattle virus 2-ferredoxinthioredoxin reductase variable subunit. Means \pm SEs, $n=3, *$ significant differences at $P<0.05$.

A previous study suggested that FTRv is involved in stress response (Keryer et al. 2004). After infection with Agrobacterium containing pTRV2-FTRv and pTRV2, the plants were subjected to low temperatures $\left(12^{\circ} \mathrm{C}\right)$. Compared with control plants, the young leaves in the PhFTRv-silenced plants were wrinkled, and distinctive necrotic lesions appeared after 3 weeks in mature leaves in PhFTRv-silenced plants grown at $12{ }^{\circ} \mathrm{C}$ (Fig. $\left.3 E-H, K, L\right)$, which was not observed at $24^{\circ} \mathrm{C}$ (Fig. $\left.3 A-D\right)$. In addition, the electrolyte leakage from cells of the PhFTRv-silenced leaves was significantly increased compared with that 
in control leaves (Fig. 3 Suppl.). These results showed that PhFTRv silencing resulted in the reduction of cold tolerance in the plants.

\section{Discussion}

The protein sequence alignments of the FTRvs showed that the carboxyl-termini had very high homology, which suggested that the carboxyl-terminus of FTRv might play an important role. Phylogenetic analysis showed that the small FTRv family has different copy numbers in different plant species. Petunia and other species of the Solanaceae family possess one copy of FTRv, while most monocot species contain two members. The present work involved the isolation of PhFTRv from petunia and the identification of its function.

Previous studies have shown that FTR functions in chloroplasts (Yoshida and Hisabori 2017). PhFTRv transcription was high in leaves and stems, where there are many chloroplasts in the cells, showing that the expression of FTR $v$ genes is consistent with their function and characteristics.

In A. thaliana, there are two FTRv isoforms, AtFTRA1 and AtFTRA2, and the single AtFTRA1 mutant exhibited phenotypic perturbations when compared with wild-type plants (Keryer et al. 2004). There is only one FTRv gene, named PhFTRv, in petunia. In petunia, PhFTRv silencing reduced chlorophyll content in leaves and delayed flowering. FTR converts a light-activated electron signal into a thiol signal that is further transmitted by Trxs. The Trx system regulates carbon flow and other biochemical processes (Schurmann and Buchanan 2008). Reduced activity of the FTR system down-regulated $\mathrm{CO}_{2}$ fixation (Buchanan 2016, Nikkanen et al. 2016). Due to the reduced $\mathrm{CO}_{2}$ fixation, the whole photosynthetic activity including photochemical reactions can be affected. In addition, a variegated leaves have been observed in the young FTRcsilenced $A$. thaliana and inapl (ftrc) mutant plants, and the sectors near the petioles of young leaves in FTRc-silenced plants appeared to be conspicuously chlorotic (Wang et al. 2014), although the characterized T-DNA insertional ftrc mutant showed no apparent physiological abnormalities due to the leaky FTR protein expression (Yoshida and Hisabori 2017).

In A. thaliana inapl ( $f t r c)$ mutants, there are pale green areas in leaves that have abnormally small mesophyll cells and reduced numbers of chloroplasts (Hashida et al. 2018). Similarly, in this study, PhFTRv silencing resulted in the reduction of the number of chloroplasts in mesophyll cells (Fig. 2 Suppl.). Wang et al. (2014) suggested that $A$. thaliana FTRc probably participates in the regulation of chloroplast development. In addition, in Pisum sativum, a phenotype characterized by palegreen leaves was observed in plants with VIGS-mediated silencing $T R X-M$ and $T R X-F$, and TRX-F regulates the ATPase activity of the magnesium chelatase CHLI subunit (Luo et al. 2012). Overall, the decrease in chlorophyll content in PhFTRv-silenced plants may be attributed to the change in the number and development of chloroplasts and/or the ATPase activity of the magnesium chelatase CHLI subunit. In addition, PhFTR $v$ silencing significantly decreased the $\mathrm{P}_{\mathrm{N}}$ and $\mathrm{g}_{\mathrm{s}}$ and significantly increased the $\mathrm{c}_{\mathrm{i}}$ in leaves, indicating the significance of chloroplast thioredoxin systems in maintaining photosynthetic efficiency in petunia.

In tomato, distinctive necrotic lesions appear after 3 weeks in $S l F T R c$-silenced plants cultured at $20^{\circ} \mathrm{C}$, while necrotic lesions in SIFTRc-silenced plants are completely suppressed at $26{ }^{\circ} \mathrm{C}$ (Lim et al. 2010). Similarly, in this study, PhFTRv silencing resulted in distinctive necrotic lesions in leaves under low temperature conditions at $12^{\circ} \mathrm{C}$, while there were no necrotic lesions in PhFTRv-silenced leaves at $24{ }^{\circ} \mathrm{C}$. It is worth noting that petunia is more resistant to cold than tomato. In addition, the expression of PhFTRv was increased under low temperature (Fig. 2B), and PhFTRv silencing resulted in wrinkled, distinctive necrotic lesions and higher electrolyte leakage from cells leaves. It seems that PhFTRv functions as a regulator of low temperature resistance in petunia plants. In addition, PhFTRv silencing could decrease the activity of Calvin cycle (Balsera et al. 2013, Buchanan 2016, Nikkanen et al. 2016), which results in lower production of sugars that can affect the response to low temperature. In $A$. thaliana, the overexpression of NTRC resulted in enhanced tolerance to heat shock, whereas NTRC knockout mutant plants exhibited a temperature-sensitive phenotype (Chae et al. 2013). Core chloroplastic TRXs (TRXf and TRXm) exhibit a slight tendency towards decreased expression in response to almost all stressors, including cold stress (Belin et al. 2015). Previous studies suggested that SlFTRc acts as a regulator of programmed cell death and pathogen resistance in tomato plants (Lim et al. 2010). Moreover, previously characterized oxidative-stresssensitive $A$. thaliana plants with FTRA mutations appear normal until they are exposed to stress conditions (Keryer et al. 2004). These results show that the TRX system is associated with many forms of stress.

In this study, PhFTRv silencing resulted in larger leaves and epidermal cells compared with the control (Fig. 3I,J and Fig. 2 Suppl.) and lower activity of Calvin cycle also can indirectly effect developmental processes including the size of leaves. Together with the effects of PhFTRv silencing on leaf pigments and division of chloroplast, these results show that PhFTR activity affects leaf development. However, there were no obvious differences in leaf morphology between the control and SlFTRc-silenced plants in tomato (Lim et al. 2010). These results could indicate the different functions of PhFTRv, the variable subunit, and SIFTRc, the catalytic subunit.

\section{References}

Balsera, M., Uberegui, E., Susanti, D., Schmitz, R.A., Mukhopadhyay, B., Schurmann, P., Buchanan, B.B.: Ferredoxin: thioredoxin reductase (FTR) links the regulation of oxygenic photosynthesis to deeply rooted bacteria. - Planta 237: 619-635, 2013.

Belin, C., Bashandy, T., Cela, J., Delorme-Hinoux, V., Riondet, C., Reichheld, J.P.: A comprehensive study of thiol reduction 
gene expression under stress conditions in Arabidopsis thaliana. - Plant Cell Environ. 38: 299-314, 2015.

Buchanan, B.B.: The path to thioredoxin and redox regulation in chloroplasts. - Annu. Rev. Plant Biol. 67: 1-24, 2016.

Buchanan, B.B., Schurmann, P., Wolosiuk, R.A., Jacquot, J.P.: The ferredoxin/thioredoxin system: from discovery to molecular structures and beyond. - Photosynth. Res. 73: 215222, 2002.

Chae, H.B., Moon, J.C., Mi, R.S., Yong H.C., Jung, Y.J., Sun, Y.L., Nawkar, G.M.: Thioredoxin reductase type C (NTRC) orchestrates enhanced thermotolerance to Arabidopsis by its redox-dependent holdase chaperone function. - Mol. Plant. 6: 323-336, 2013.

Chang, M.M., Li, A., Feissner, R., Ahmad, T.: RT-qPCR demonstrates light-dependent AtRBCS1A and AtRBCS3B mRNA expressions in Arabidopsis thaliana leaves. - Biochem. Mol. Biol. Edu. 44: 405-411, 2016.

Chen, G., Liu, H., Wei, Q., Zhao, H., Liu, J., Yu, Y.: The acylactivating enzyme PhAAE13 is an alternative enzymatic source of precursors for anthocyanin biosynthesis in petunia flowers. - J. exp. Bot. 68: 457-467, 2017.

Dai, S., Schwendtmayer, C., Schuermann, P., Ramaswamy, S., Eklund, H.: Redox signaling in chloroplasts: cleavage of disulfides by an iron-sulfur cluster. - Science 287: 655-658, 2000 .

Dietz, K., Pfannschmidt, T.: Novel regulators in photosynthetic redox control of plant metabolism and gene expression. Plant Physiol. 155: 1477-1485, 2011.

Geigenberger, P., Fernie, A.: Metabolic control of redox and redox control of metabolism in plants. - Antioxid. Redox Signal. 21: 1389-1421, 2014.

Hashida, S., Miyagi, A., Nishiyama, M., Yoshida, K., Hisabori, T., Kawai-Yamada, M.: Ferredoxin/thioredoxin system plays an important role in the chloroplastic NADP status of Arabidopsis. - Plant J. 95: 947-960, 2018.

He, B., Gu, M., Wang, X., He, X.: The effects of lead on photosynthetic performance of waxberry seedlings (Myrica rubra). - Photosynthetica 56: 1147-1153, 2018.

Keryer, E., Collin, V., Lavergne, D., Lemaire, S., IssakidisBourguet, E.: Characterization of Arabidopsis mutants for the variable subunit of ferredoxin:thioredoxin reductase. Photosynth. Res. 79: 265-274, 2004.

Lichtenthaler, H.K.: Chlorophylls and carotenoids: pigments of photosynthetic biomembranes. - Method Enzymol. 148C: 350-382, 1987.

Lim, C.J., Kim, W.B., Lee, B., Lee, H.Y., Kwon, T., Park, J.M., Kwon, S.: Silencing of SIFTR-c, the catalytic subunit of ferredoxin: thioredoxin reductase, induces pathogenesisrelated genes and pathogen resistance in tomato plants. -
Biochem. biophys. Res. Commun. 399: 750-754, 2010.

Liu, J., Chang, X., Ding, B., Zhong, S., Peng, L., Wei, Q., Meng, J., Yu, Y.: PhDHS is involved in chloroplast development in Petunia. - Front. Plant Sci. 10: 284, 2019

Liu, J., Li, J., Wang, H., Fu, Z., Liu, J., Yu, Y.: Identification and expression analysis of $E R F$ transcription factor genes in petunia during flower senescence and in response to hormone treatments. - J. exp. Bot. 62: 825-840, 2011.

Liu, Y., Schiff, M., Dinesh-Kumar, S.P.: Virus-induced gene silencing in tomato. - Plant J. 31: 777-786, 2002.

Livak, K.J., Schmittgen, T.D.: Analysis of relative gene expression data using real-time quantitative PCR and the 2-DELTADELTACT method. - Methods 25: 402-408, 2001.

Luo, T., Fan, T., Liu, Y., Rothbart, M., Yu, J., Zhou, S., Grimm, B., Luo M.: Thioredoxin redox regulates ATPase activity of magnesium chelatase CHLI subunit and modulates redox-mediated signaling in tetrapyrrole biosynthesis and homeostasis of reactive oxygen species in pea plants. - Plant Physiol. 159: 118-130, 2012.

Nikkanen, L., Toivola, J., Rintamaki, E.: Crosstalk between chloroplast thioredoxin systems in regulation of photosynthesis. - Plant Cell Environ. 39: 1691-1705, 2016.

Sakamoto, W., Zaltsman, A., Adam, Z., Takahashi, Y.: Coordinated regulation and complex formation of Yellow Variegated1 and Yellow Variegated2, chloroplastic FtsH metalloproteases involved in the repair cycle of photosystem II in Arabidopsis thylakoid membranes. - Plant Cell 15: 2843 $2855,2003$.

Schurmann, P., Buchanan, B.B.: The ferredoxin/thioredoxin system of oxygenic photosynthesis. - Antioxid. Redox Signal. 10: 1235-1274, 2008.

Schurmann, P., Jacquot, J.P.: Plant thioredoxin systems revisited. - Annu. Rev. Plant Physiol. 51: 371-400, 2000.

Serrato, A.J., Pérez-Ruiz, J.M., Spínola, M.C., Cejudo, F.J.: A novel NADPH thioredoxin reductase, localized in the chloroplast, which deficiency causes hypersensitivity to abiotic stress in Arabidopsis thaliana. - J. biol. Chem. 279 : 43821-43827, 2004.

Wang, P., Liu, J., Liu, B., Da, Q., Feng, D., Su, J., Zhang, Y., Wang, J., Wang, H.: Ferredoxin: thioredoxin reductase is required for proper chloroplast development and is involved in the regulation of plastid gene expression in Arabidopsis thaliana. - Mol. Plants 7: 1586-1590, 2014.

Yoshida, K., Hisabori, T.: Distinct electron transfer from ferredoxin-thioredoxin reductase to multiple thioredoxin isoforms in chloroplasts. - Biochem. J. 474: 1347-1360, 2017.

Yoshida, K., Noguchi, K., Motohashi, K., Hisabori, T.: Systematic exploration of thioredoxin target proteins in plant mitochondria. - Plant Cell Physiol. 54: 875-892, 2013. 